\title{
Computer-aided Prognosis of Neuroblastoma: Detection of Mitosis and Karyorrhexis Cells in Digitized Histological Images
}

\author{
Olcay Sertel, Student Member, IEEE, Umit V. Catalyurek, Member, IEEE, Hiroyuki Shimada, and \\ Metin N. Gurcan, Senior Member, IEEE
}

\begin{abstract}
Histopathological examination is one of the most important steps in evaluating prognosis of patients with neuroblastoma (NB). NB is a pediatric tumor of sympathetic nervous system and current evaluation of NB tumor histology is done according to the International Neuroblastoma Pathology Classification. The number of cells undergoing either mitosis or karyorrhexis (MK) plays an important role in this classification system. However, manual counting of such cells is tedious and subject to considerable inter- and intra-reader variations. A computer-assisted system may allow more precise results leading to more accurate prognosis in clinical practice. In this study, we propose an image analysis approach that operates on digitized NB histology samples. Based on the likelihood functions estimated from the samples of manually marked regions, we compute the probability map that indicates how likely a pixel belongs to an MK cell. Component-wise 2-step thresholding of the generated probability map provides promising results in detecting MK cells with an average sensitivity of $81.1 \%$ and 12.2 false positive detections on average.
\end{abstract}

\section{INTRODUCTION}

$\mathrm{N}$ EUROBLASTOMA (NB) is a pediatric tumor of sympathetic nervous system. It is the most common cancer in infants and approximately 650 patients are diagnosed with NB each year in the United States [1]. One of the most important indicators for predicting clinical behavior and prognosis of patients with this disease is the histological examination of biopsy/surgical samples. The World Health Organization recommends the use of the International Neuroblastoma Pathology Classification for categorization of the NB into different prognostic groups [2, 3]. According to this classification system, tumor histology is characterized as either "favorable" or "unfavorable" based on age-linked evaluation of morphology.

One of the most critical steps in histological evaluation is

Manuscript received April 7, 2009. This work was supported in part by the Young Investigator Award from the Children's Neuroblastoma Cancer Foundation.

O. S. is with the Department of Electrical and Computer Engineering and the Department of Biomedical Informatics, The Ohio State Univ., Columbus, OH, 43210, USA (corresponding author, phone: 614-292-5970; fax: 614-688-6600; e-mail: osertel@ bmi.osu.edu).

U. V. C. is with Department of Electrical and Computer Engineering and the Department of Biomedical Informatics, The Ohio State University, Columbus, OH, 43210 USA (e-mail: umit@ bmi.osu.edu).

H. S. is with the Department of Pathology and Laboratory Medicine, Los Angeles Children's Hospital, Los Angeles, CA, 90027, USA (email: HShimada@chla.usc.edu).

M. N. G. is with the Department of Biomedical Informatics, The Ohio State University, Columbus, OH, 43210 USA (e-mail: metin.gurcan@ osumc.edu). to determine the mitosis-karyorrhexis index (MKI) [3]. MKI is defined as the number of tumor cells either in the process of mitosis or karyorrhexis. Mitosis is the division of a cell into two cells, whereas karyorrhexis is the destructive fragmentation of the nucleus of a dying cell. A higher MKI usually indicates a higher likelihood for the tumor to be unfavorable. It is reported that $\mathrm{MYCN}$ oncogene amplification, a molecular indicator for predicting aggressive tumor progression, is a powerful driving force to increase mitotic and karyorrhectic (MK) activities in NB tumors $[2,3]$.

In clinical practice, MKI is determined after a tedious microscopic examination of hematoxylin and eosin (H\&E) stained tissue slides at high magnifications. The pathologists examine areas within the slide and count the number of $\mathrm{MK}$ cells among 5000 cells. This process is cumbersome and often subject to sampling bias due to enormous size of the images (up to $120 \mathrm{k} \times 100 \mathrm{k}$ resolution). This results in considerable inter- and intra-reader variations of up to $20 \%$ between central and institutional reviewers in NB prognosis [4].

Histopathological image analysis is an emerging field and it becoming increasingly popular mostly due to the recent developments in the scanning technology, which made it possible to digitize the whole tissue slides at high magnifications. However, there are several challenges ahead. The variations between samples of the same cancer type, either due to relatively distinct content or due to the slide preparation stages, make it difficult to develop adaptive and robust algorithms. Nevertheless, there are a number of computerized systems developed for several cancer types such as follicular lymphoma [5], prostate cancer [6], and breast cancer [7].

In our previous work for NB prognosis, we also developed computerized systems to classify grade of differentiation and stromal development [8, 9]. These systems operate in a multi-resolution manner and at each resolution, perform segmentation based on color and spatial information and classification based on texture of the particular cytological components in the tissue. The promising results reported in these studies showed that it is possible to extract objective and precise diagnostic clues that will lead to precise histopathological evaluations of tissue samples. The MKI component being presented in this study will also be incorporated to the existing systems for the complete NB Virtual Shimada Classification System. A cell 
segmentation method has also been proposed, but this is not designed to detect MK cells [10].

\section{DETECTION OF MITOSIS \& KARYORRHEXIS CELLS}

The inputs to our system are H\&E-stained tissue samples digitized at 40x using a ScanScope T2 digitizer (Aperio, San Diego, CA). Typically, the resulting digital images have very large resolutions up to $120 \mathrm{~K} \times 100 \mathrm{~K}$. To make the training and evaluation feasible, we manually cropped ten regions of interest (ROI) images associated with different NB subtypes, i.e., low MKI (less than 100 cells in MK cells among 5000 cells on average), intermediate MKI (between 100 and 200 MK cells) and high MKI (more than 200 MK cells). An expert pathologist verified the content of these ROIs and manually annotated the MK cells in each ROI image of $888 \times 1472$ pixels resolution. These manual markings established the ground-truth information and we used this information to evaluate the performance of the proposed system. Fig. 1 shows some example image regions in which MK cells are circled.

Providing a good contrast between cytological structures at cellular level, hematoxylin and eosin (H\&E) is one of the most widely used staining methods in histology. It dyes the nuclear structures with blue-purple hue and protein rich regions, such as cytoplasm and extracellular material, with bright pink hue. Biologically, MK figures are characterized by condensed nuclear material; hence such regions are intensively stained with hematoxylin, therefore appearing in the slides as very dark blue-purple color (Fig. 1). Mitotic figures are more or less rod-shaped with spiked projections and absence of a nuclear membrane, whereas karyorrhectic cells have fragmented nuclear material usually accompanied by condensed eosinophilic cytoplasm. For NB prognosis purposes, there is no need to distinguish between mitotic and karyorrhectic cells; hence they are detected as one category, i.e., MK.

We used a probabilistic approach for the MK cell detection problem. We first applied a pre-processing step to bring the dynamic color range of the sample images in the database into a common distribution. Then, using samples in the training set, we estimated the likelihood functions for pixel intensities that correspond to MK cells. Using these likelihood functions, we computed the probability maps, in which a high probability indicates a higher chance of belonging to an MK cell. Finally, using a 2-step componentwise thresholding, we detected the candidate MK cells. Following subsections provide more detailed explanations of these steps.

\section{A. Image Preprocessing}

The staining conditions of the slides vary considerably from one slide to another. Hence, after the digitization step, images may have quite different dynamic ranges. We used histogram equalization to normalize the color distribution across slides with different staining conditions [11]. An image with ideal staining conditions was determined and the
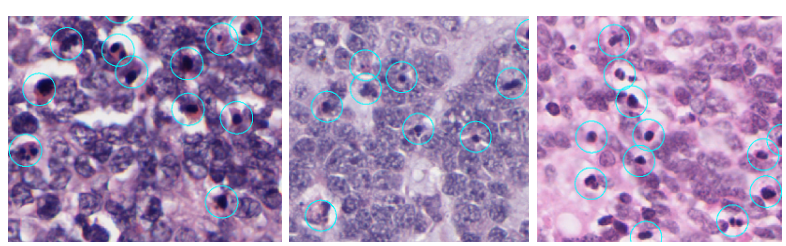

Fig. 1. Sample image regions, where MKI cells circled. Note the wide variation in color information due to the staining differences.

dataset was normalized to that color distribution.

After histogram equalization, we applied the anisotropic diffusion to smooth the relatively heterogeneous regions, while preserving important edge information [12]. Anisotropic diffusion is an iterative smoothing process and characterized as follows:

$$
I(x, y)_{s+1}=I(x, y)_{s}+\lambda C(x, y)_{s}^{T} \nabla I(x, y)_{s}
$$

where $I \in\{R, G, B\}$ color channels, and $I(x, y)_{s}$ is the color channel at step $s$. $\lambda$ is a constant that needs to satisfy $0 \leq \lambda \leq 0.25$ when numerical stability is sustained. $\nabla I(x, y)_{s}=\left(\nabla I\left(x_{-}, y\right)_{s}, \nabla I\left(x_{+}, y\right)_{s}, \nabla I\left(x, y_{+}\right)_{s}, I\left(x, y_{-}\right)_{s}\right)^{T}$ is a vector of nearest neighbor differences. It is used to update the conduction coefficient vector $C(x, y)_{s}=\left(c\left(x_{-}, y\right)_{s}, c\left(x_{+}, y\right)_{s}, c\left(x, y_{+}\right)_{s}, c\left(x, y_{-}\right)_{s}\right)^{T} \quad$ at each step:

$$
c(u, v)_{s}=g\left(\nabla I(u, v)_{s}\right), \text { where } g(\nabla I)=\frac{1}{1+(\|\nabla I\| / \kappa)^{2}} .
$$

In our application, we experimentally determined $\lambda=0.2, \kappa=10$.

\section{B. Computing the Likelihoods}

For the detection of MKI cells, we used the Bayesian decision framework, which relies on Bayes' law for conditional probability:

$$
P\left(\omega_{j} \mid X\right)=\frac{p\left(X \mid \omega_{j}\right) P\left(\omega_{j}\right)}{p(X)}
$$

where $X$ is the feature vector consisting of color information in the red-green-blue (RGB) color space for each pixel, and $\omega_{j}, j=\{0,1\}$ indicates either a pixel belongs to MKI or not, respectively. $P\left(\omega_{j} \mid X\right)$ is the posterior probability that a pixel belongs to class $\omega_{j}$ given features $X, p\left(X \mid \omega_{j}\right)$ is the likelihood that a sample of class $\omega_{j}$ exhibits features $x . P\left(\omega_{j}\right)$ is the prior probability of class $\omega_{j}$, and $p(X)$ is the evidence factor, and can be viewed as a scale factor that guarantees that the posterior probabilities sum to one [13].

In fact, the evidence term, $p(X)$, is independent of class membership. Furthermore, we assume that a priori 


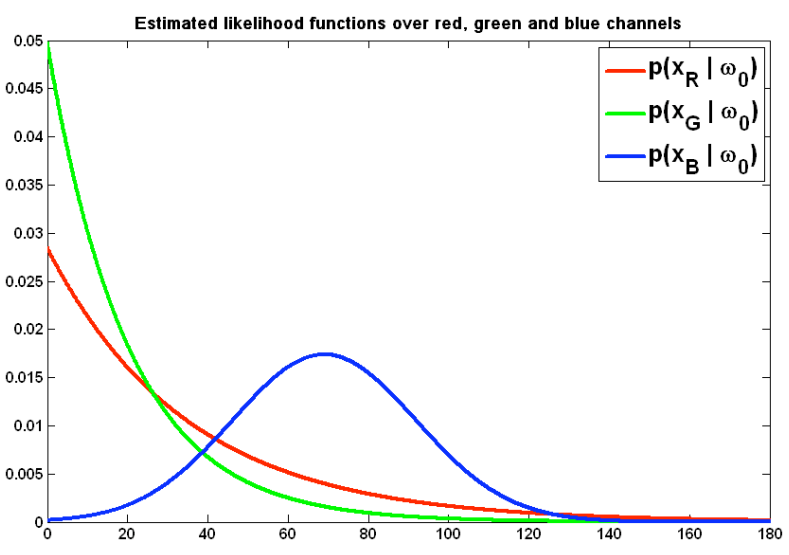

Fig. 2. Likelihood functions are estimated from red, green and blue channels using the manually marked samples.

probabilities (i.e., probability of a pixel being part of a cell that is in MK or not) are equally likely. Then, the posterior probability simplifies to the likelihood term. Under the assumption of independent features (i.e. the RGB color values), the posterior probability simply becomes:

$$
p\left(\omega_{j} \mid X\right)=\prod_{i=R, G, B} p\left(x_{i} \mid \omega_{j}\right)
$$

The likelihood functions $p\left(x_{i \in R, G, B} \mid \omega_{0}\right)$, for MK cells are constructed by manually sampling such pixels. The underlying distributions of these training pixels are estimated using maximum likelihood estimation [13]. The resulting likelihood functions for MKI cells are given in Fig. 2. The posterior probability $p\left(x_{i} \mid \omega_{0}\right)$ for MKI cells is further normalized to $[0,1]$ before the thresholding step.

\section{2-Step Component-wise thresholding}

Once we computed the MKI likelihood, $p\left(\omega_{0} \mid X\right)$, we applied a 2-step component-wise thresholding to obtain candidate $\mathrm{MK}$ cell regions. The first step is regular thresholding using $\tau_{1}=0.1$ over the posterior probability map. This is a very low threshold, allowing too many false positives, yet including almost all of the significant MK cells. In the second step, we first computed the connected components and calculated the mean probability for every region. Using the second threshold $\tau_{2}=0.25$, most of the false positive detections are removed.

Fig. 3 illustrates the idea behind this thresholding approach for a 1-dimensional case. Experimentally, we determined that most of the false positive detections have gradual decrease in the probability map around regions with high MK likelihood, whereas around real MK cells, we observe sharp, step like changes. In this scenario, it is not possible to detect the real MK case while avoiding the false positive detection, using for example hysteresis thresholding [10]. However, the proposed component-wise 2-step thresholding allows being highly sensitive in the first step while ensuring the specificity in the second step. Fig. 4

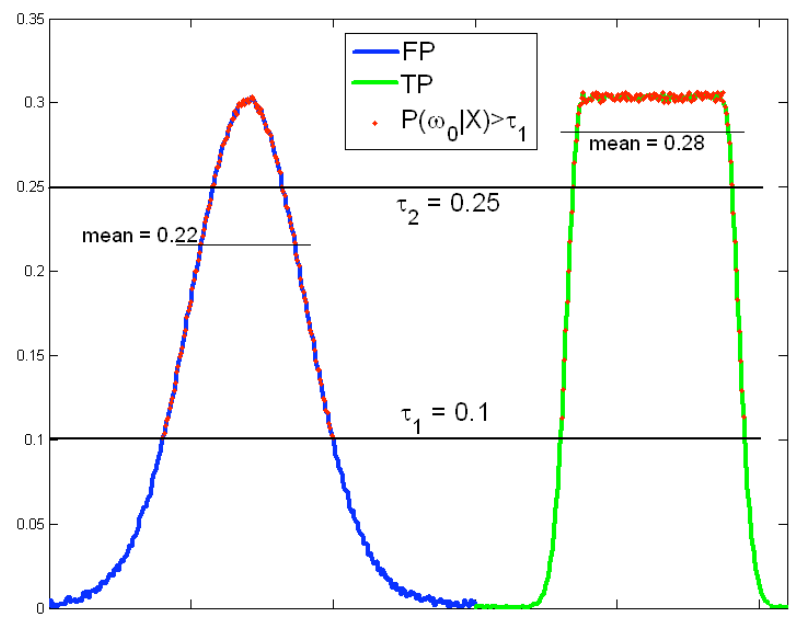

Fig. 3. Illustration of the component-wise 2-step threshold for 1dimensional case. $\tau_{1}$ provides the connected components and $\tau_{2}$ thresholds the mean probability of each component.

shows the intermediate results on a sample ROI image.

\section{EXPERIMENTAL RESULTS}

We applied the proposed approach to ten representative ROI images extracted from NB whole-slide images. Four of these images are cropped from low (images 1-4), four from intermediate (images 5-8), and two from high (images 9,10) MK subtypes. Computerized results have been compared to the ground truth information provided by an expert pathologist. The corresponding number of true positive (TP), false positive (FP), ground-truth (GT) cells as well as the detection sensitivity (i.e., TP/GT) are reported in Table 1 . It should be noted that MKI classification is based on MK count among 5000 cells and these sample ROIs do not exhibit same cellularity; hence their ground-truths are based on the whole-slides they are cropped from.

As can be seen from Table 1, the proposed computerized system was able to detect, on average, 55 out of 68 cells, thus providing a detection rate of the $81.1 \%$. The average number of false positives was 12.2 cells that correspond to approximately $18 \%$ of sensitivity, which is acceptable especially given the heterogeneity of the tissue content and variations among different NB subtypes.

TABLE I

MK CELL DETECTION RESULTS OVER 10 REPRESENTATIVE ROI. GT, TP, FP CORRESPOND TO THE NUMBER OF GROUND-TRUTH, TRUE POSITIVE, AND FALSE POSITIVE MK CELLS, RESPECTIVELY

\begin{tabular}{lrrrr}
\hline \hline Image & \multirow{2}{*}{ GT } & \multicolumn{2}{c}{ TP } & \multicolumn{2}{l}{ FP } & \multicolumn{1}{c}{ Sensitivity } \\
\hline 1 & 12 & 9 & 10 & $75.0 \%$ \\
2 & 15 & 12 & 12 & $80.0 \%$ \\
3 & 44 & 40 & 16 & $90.9 \%$ \\
4 & 37 & 36 & 13 & $97.3 \%$ \\
5 & 44 & 39 & 16 & $88.6 \%$ \\
6 & 37 & 31 & 13 & $83.8 \%$ \\
7 & 73 & 65 & 8 & $89.1 \%$ \\
8 & 45 & 38 & 3 & $84.4 \%$ \\
9 & 150 & 102 & 14 & $68.0 \%$ \\
10 & 221 & 178 & 17 & $80.5 \%$ \\
Avg. & $\mathbf{6 7 . 8}$ & $\mathbf{5 5 . 0}$ & $\mathbf{1 2 . 2}$ & $\mathbf{8 1 . 1 \%}$
\end{tabular}




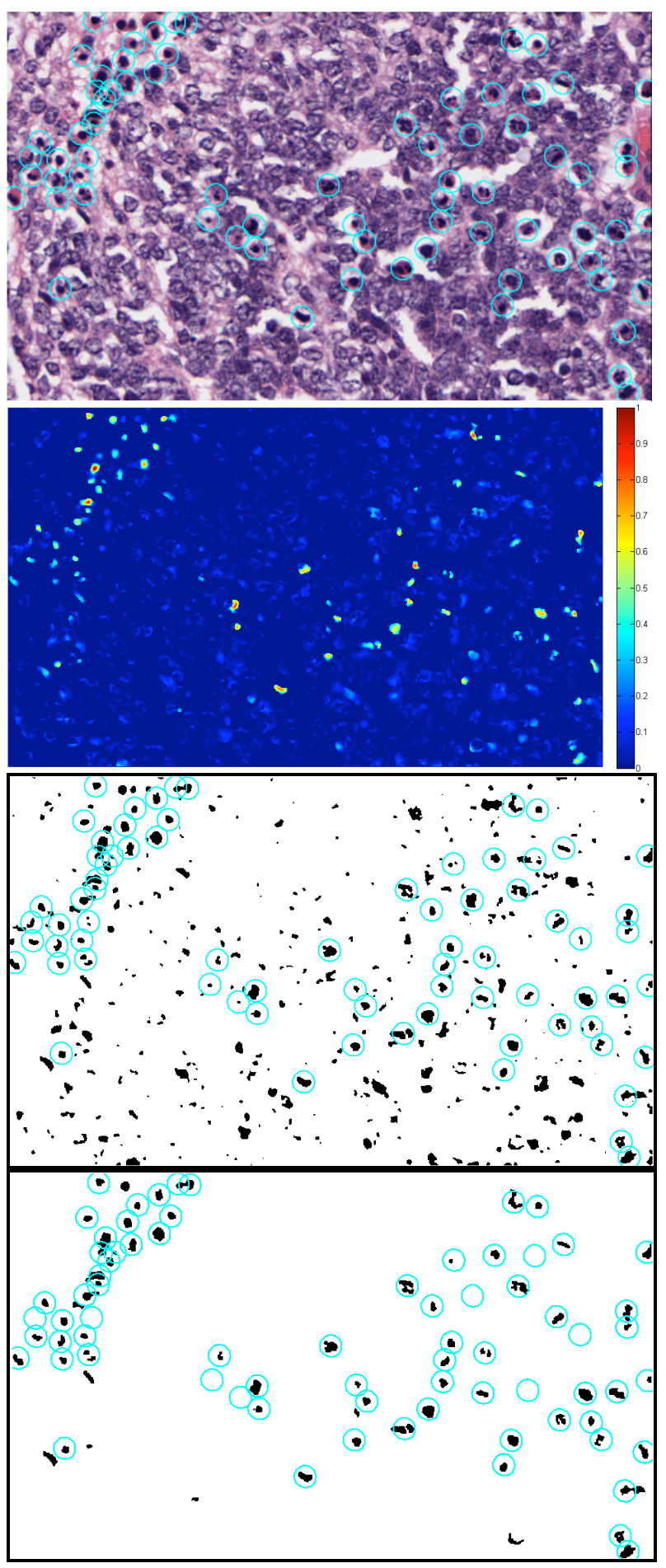

Fig. 4. Intermediate results of the computerized MKI detection approach are shown above. (a) Original input image with ground-truth shown in cyan circles (b) probability map $p\left(\omega_{0} \mid X\right)$ of MKI cells, (c) results of the first thresholding, $p\left(\omega_{0} \mid X\right)>\tau_{1}$ (d) results after applying the component-wise second threshold $p_{a v g}^{\text {comp }}\left(\omega_{0} \mid X\right)>\tau_{2}$.

\section{CONCLUSIONS}

In this study, a computer-aided system based on the likelihood functions and 2-step component-based thresholding was developed for the automated detection of MK cells in digitized images of NB tissue slides. Each NB image is first preprocessed to reduce the variation in the color range due to staining differences and to smooth the highly heterogeneous regions. Candidate $\mathrm{MK}$ cell regions were then determined based on the likelihood functions. Finally, the MKI cells were detected by applying a component-wise 2-step thresholding step. The computerized results were compared to ground-truth information provided by an expert pathologist. The proposed approach yielded promising results with $81.1 \%$ of detection rate and 12.2 false positives on average over a testing set of ten images. In our future work, we are going to combine this system with the previously developed components to obtain a complete computer-assisted NB prognosis system, which will be useful in clinical settings.

\section{REFERENCES}

[1] American Cancer Society, http://www.cancer.org (2007).

[2] H. Shimada, et al., "The international neuroblastoma pathology classification", Cancer, Vol. 86, No. 2, pp. 364-372, 1999

[3] H. Shimada, et al., "Terminology and morphologic criteria of neuroblastic tumors", Cancer, Vol. 86, No. 2, pp. 349-363, 1999.

[4] L. A. Teot, et al., 'The problem and promise of central pathology review", Pediatric and Developmental Pathology, Vol. 10 pp. 199207, 2007.

[5] O. Sertel, et al. "Histopathological Image Analysis Using ModelBased Intermediate Representations and Color Texture", J. of Signal Proc. Sys., In Press, 2009. (doi: 10.1007/s11265-008-0201-y).

[6] A. Tabesh, et al., "Multifeature prostate cancer diagnosis and Gleason grading of histological images," IEEE Trans. on Medical Imaging, vol. 26, pp. 1366-1378, 2007.

[7] S. Doyle, et al., "Automated Grading of Breast Cancer Histopathology Using Spectral Clustering ", Proc. of IEEE Int. Symp. on Biomedical Imaging, pp. 496-499, 2008.

[8] O. Sertel, et al., "Computer-aided prognosis of neuroblastoma on whole-slide images: Classification of stromal development”, Pattern Recognition, In Press, (doi:10.1016/j.patcog.2008.08.027).

[9] J. Kong, et al., "Computer-aided grading of neuroblastic differentiation”, Proc. of IEEE Int. Conf. on Image Processing, San Antonio, TX, pp. 525-528,Sept., 2007.

[10] M. N. Gurcan, et al., "Image Analysis for neuroblastoma classification: Segmentation of cell nuclei”, IEEE Int. Conf. of Eng. In Medicine and Biology, Sept., New York City, 2006.

[11] R. C. Gonzales, and R. E. Woods, Digital Image Processing, Addison-Wesley Publishing Co., 1993.

[12] P. Perona, and J. Malik, "Scale-space and edge detection using ansotropic diffusion", IEEE Trans. on PAMI, Vol. 12, No. 7, pp. 629 $639,1990$.

[13] R. O. Duda, P. E. Hart, and D. G. Stork, Pattern Classification, Wiley-Interscience Publication, 2001 